\title{
EL TRABAJO POR PROYECTOS EN EL CURRÍCULO EDUCATIVO: PRINCIPIOS METODOLÓGICOS QUE LO FUNDAMENTAN
}

\author{
Deilis Ivonne Pacheco Sanz \\ Universidad de Valladolid \\ deilisivonne.pacheco@uva.es \\ Alejandro Canedo García \\ Universidad de León \\ acang@unileon.es \\ Beatriz Martín Sánchez \\ Universidad de Valladolid \\ beamarsan7@hotmail.com \\ Yvet Bleye Varona \\ Universidad de Valladolid \\ yvet.b@hotmail.com \\ Angela Gago Morate \\ Universidad de Valladolid \\ agagomorate@gmail.com
}

Recepción Artículo: 6 febrero 2020

Admisión Evaluación: 4 marzo 2020

Informe Evaluador 1: 1 enero 2020

Informe Evaluador 2: 1 enero 2020

Aprobación Publicación: 20 abril 2020

\section{RESUMEN}

Aunque desde años atrás, pedagogos/as y maestros/as han defendido metodologías más innovadoras que las que se estaban desarrollando en las escuelas, es, en este momento, cuando de manera más ferviente, se está removiendo algo en gran parte del profesorado que está llevando a querer cambiar los procesos de enseñanzaaprendizaje actuales, queriendo innovar y probando nuevos enfoques pedagógicos. El trabajo por proyectos (TP) es un claro ejemplo de esas nuevas perspectivas pedagógicas que se están ejecutando cada vez más en las aulas. Dar fundamento teórico a esta metodología activa y de innovación, sustentar teóricamente la práctica docente en torno al trabajo por proyectos y proponer un modelo de intervención a partir de los principios metodológicos que lo fundamentan, son los objetivos generales o resultados que se esperan al finalizar el proceso de revisión de la evidencia disponible, sobre la relación entre los principios metodológicos que orientan la práctica docente para el desarrollo del Trabajo por Proyectos (construcción de aprendizajes significativos, funcionalidad del aprendizaje, capacidad de aprender a aprender, modificación de los esquemas de conocimiento que el alumno/a ya posee, actividad intensa por parte del alumno/a -observación, experimentación, investigación-, individualización, socialización, autoconcepto positivo, motivación, juego, globalización) y el éxito del proceso de enseñanza-aprendi- 


\section{EL TRABAJO POR PROYECTOS EN EL CURRÍCULO EDUCATIVO: PRINCIPIOS METODOLÓGICOS QUE LO FUNDAMENTAN}

zaje. Se concentró la búsqueda en artículos publicados entre 1998 y 2017, los cuales se sintetizan en una tabla en la que se examinan intervenciones que se han realizado con el objetivo de promover el TP con la finalidad ulterior de que el mismo mejore el proceso de enseñanza-aprendizaje. El cuerpo de evidencias revisado arroja resultados de intervenciones que han comprobado ser efectivas en promover el TP dentro del currículo de cualquier nivel/modalidad educativa, sugiriendo que existe una relación de causalidad donde el TP predice el éxito del proceso educativo (Se recibieron fondos de la MOVILIDAD INVESTIGADORES Uva BANCO SANTANDER 2020).

Palabras clave: trabajo por proyectos; principios metodológicos; éxito; proceso de enseñanza-aprendizaje; modelo de intervención

\section{ABSTRACT}

Project work in the educational curriculum: methodological principles that fund it. Although for years ago, pedagogues and teachers have advocated more innovative methodologies than those that were being developed in schools, it is, at this moment, when most fervently, something is being removed in a large part of the teachers that are leading to wanting to change the current teaching-learning processes, wanting to innovate and trying new pedagogical approaches. Project work (PW) is a clear example of these new pedagogical perspectives that are increasingly being implemented in classrooms. Give theoretical foundation to this active and innovative methodology, theoretically support the teaching practice around project work and propose an intervention model based on the methodological principles that support it, are the general objectives or results expected at the end of the review process of the available evidence, on the relationship between the methodological principles that guide teaching practice for the development of Project Work (construction of significant learning, learning functionality, ability to learn to learn, modification of the knowledge schemes that the student already has intense activity on the part of the student -observation, experimentation, research-, individualization, socialization, positive self-concept, motivation, play, globalization) and the success of the teaching-learning process. The search was concentrated on articles published between 1998 and 2017, which are synthesized in a table that examines interventions that have been carried out with the aim of promoting PW with the subsequent aim that it improves the teaching process- learning. The body of evidence reviewed yields results of interventions that have been proven to be effective in promoting PW within the curriculum of any educational level / modality, suggesting that there is a causal relationship where PW predicts the success of the educational process (Funds were received from the MOBILITY RESEARCHERS UVa BANCO SANTANDER 2020).

Keywords: project work; methodological principles; success; teaching-learning process; intervention model

\section{ANTECEDENTES}

Convertir el aula de clases en una comunidad de aprendizaje conlleva a abandonar el modelo fabril del pasado y permite crear espacios de comunicación y de indagación, donde se construya el conocimiento.

En la sociedad en la que nos movemos uno de los pilares fundamentales, en cualquier campo, es el saber hacer, pues ya no basta con poseer una serie de conocimientos teóricos, sino que es necesario saber llevarlos a la práctica. De aquí, el papel crucial que desempeñan las metodologías activas (presenciales y virtuales), alternativas e innovadoras en el proceso educativo, el cual debe adaptarse a las demandas planteadas por la sociedad del siglo XXI que exige cambios repentinos, constantes y progresivos de paradigmas. Ante esta situación, los actuales sistemas educativos de enseñanza no pueden quedarse de brazos cruzados, la sociedad les incita a promover cambios sustanciales en las metodologías para avanzar hacia una nueva concepción de los procesos de enseñanza-aprendizaje. El sistema educativo, de manera obligatoria, debe hacer hincapié en cuatro focos de interés: el estudiantado como protagonista del proceso, el profesorado como facilitador del aprendizaje, el contexto educativo y los programas/currículo vinculados al contexto o entorno social en el que se ejecuta el proceso educativo. Por lo tanto, este nuevo enfoque afecta de manera directa al papel del profesorado en el proceso de ense- 
ñanza-aprendizaje, a sus métodos y estrategias didácticas, a los procesos de evaluación de los aprendizajes, etc. (Pacheco, Canedo, Carpio y García, 2019).

Dentro de este marco cobra gran importancia el diseño, implementación y evaluación de diferentes metodologías didácticas de carácter activo e innovador, cuya efectividad se ha ido constatando a lo largo del tiempo. El trabajo por proyectos es una de ellas, pues son una apelación a la inventiva, la imaginación y la aventura de enseñar y aprender. Los TP rescatan la perspectiva del alumnado, presentan el conocimiento como un constructor global, rompen con la homogeneidad del libro de texto, facilitan la interconexión con la vida no-escolar y animan a la autonomía profesional de las/los docentes. Además, cuando se implementan, i) la intervención pedagógica va encaminada a promover el aprendizaje significativo del alumnado de una manera intencional y reflexiva; ii) plantean el conocimiento como una elaboración activa por parte del alumnado y no como la mera recepción pasiva de una serie de datos; iii) contemplan los contenidos como experiencias que se viven (Pacheco et al., 2019; Ureña, 2013; Mérida y Ruiz, 2016).

Es importante reafirmar la idea de que el T.P. no es una metodología didáctica, sino una concepción de la educación que no olvida que el trabajo del profesorado no es el de una transmisora o transmisor de contenidos empaquetados, sino una profesión moral.

\section{OBJETIVOS}

Dar fundamento teórico al trabajo por proyectos y proponer un modelo o secuencia metodológica para su aplicación, a partir de los principios que lo fundamentan, son los objetivos generales o resultados que se esperan al finalizar el proceso de revisión de la evidencia empírica disponible.

\section{MUESTRA}

Se recogen 14 artículos recientes, entre 2010 y 2017, producto de la búsqueda enfocada hacia la factibilidad de la aplicación del TP en las aulas educativas y como predictor de alguna medida de bienestar o éxito académico de quienes participan en el proceso de su ejecución.

\section{METOdOLOGíA}

El objetivo de este trabajo condujo a la exploración, descripción, explicación y predicción de aspectos relevantes que dieran fundamento al TP. Para ello, se elaboró una tabla tipo con la información recogida de artículos cientííicos seleccionados en bases de datos como Dialnet y Google Scholar (ver tabla 1). En dicha tabla, se examinan estudios en los que el TP es predictor de resultados que son considerados como una medida de éxito en el ambiente escolar. Por otro lado, también se accedió a estudios sobre intervenciones diseñadas para promover la factibilidad de aplicación del TP con la finalidad ulterior de que el mismo mejore el proceso de enseñanzaaprendizaje. Ambos criterios: éxito académico y factibilidad de aplicación, permitieron el análisis de los resultados y, además, detectar una serie de aspectos que convergen para caracterizar y dar sustento teórico al TP.

Asimismo, el estudio se justifica en las ideas que se expresan en la primera parte de este trabajo, las cuales subrayan que el TP es una de las metodologías que se ajusta a la sociedad actual. 
Tabla 1.Síntesis de estudios que promueven la aplicación del TP

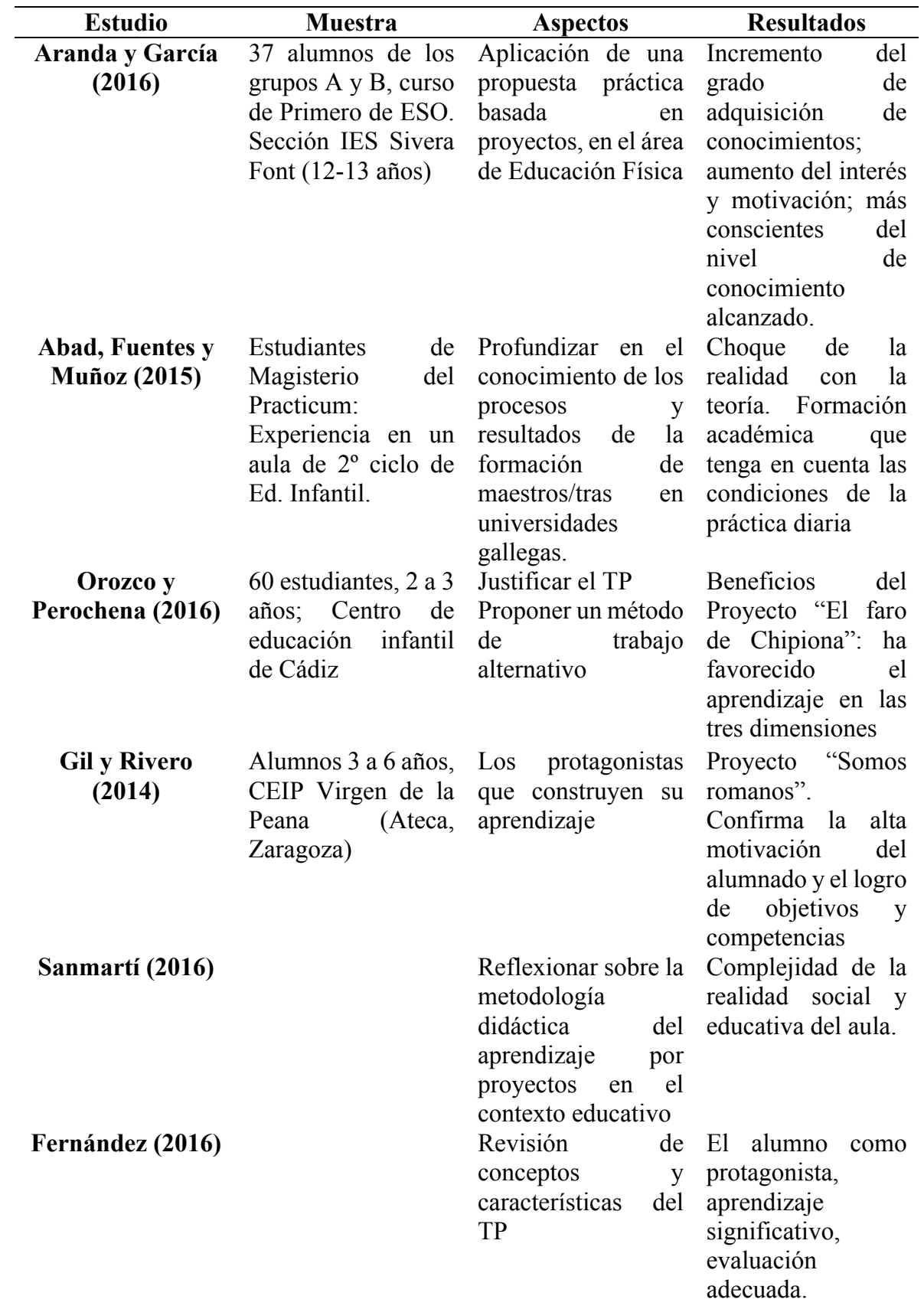


Ureña (2013) Alumnado de 5 Colaboración en la Fases de un TP: años, Colegio creación de Elección del tema. Federico García ciudadanos Conocimientos Lorca de París, competentes a previos. Qué Curso 2012/2013 través del proyecto queremos saber. "El esqueleto" Organización del trabajo.

Investigación.

Actividades.

Evaluación

Moliner y $35 \quad$ estudiantes Experiencia

Sánchez- Máster de innovadora

Tarazaga (2015) Psicopedagogía, Universidad Jaume I

Gutiérrez (2015)

Uso del TP en las

El TP permite poner de manifiesto la renuncia al sentido excluyente de la universidad como contexto formativo, ampliar $\mathrm{y}$ enriquecer las experiencias de aprendizaje, diversificar el estatus de los estudiantes

aulas de Educación Plástica y Visual Infantil deben ser fruto de la formación sistemática que proporciona la escuela, la materia artística requiere integrarse en un plan de trabajo no conductista

Mérida et al. Estudiantes y El TP para la $\mathrm{Se}$ observa la (2011) Profesores de la interacción entre necesidad de Facultad de CC. de maestras en activo y establecer redes la Educación de alumnado de formativas entre Córdoba, Maestras Magisterio, a través profesionales en de Educación de una comunidad educación y crear Infantil, de aprendizaje Comunidades de Alumnos/as de Escuela- Aprendizaje Educación Infantil y Universidad asesores del CEP 


\section{EL TRABAJO POR PROYECTOS EN EL CURRÍCULO EDUCATIVO: PRINCIPIOS METODOLÓGICOS QUE LO FUNDAMENTAN}

\section{RESULTADOS}

En esta sección, por una parte, se discuten los resultados de los artículos revisados, de acuerdo a criterios que permiten evidenciar tanto la factibilidad de la aplicación del TP (en los diferentes niveles educativos/ áreas de estudio), como su contribución al éxito del proceso de enseñanza- aprendizaje. Por otra parte, se extraen los principios metodológicos o bases ideológicas que orientan la práctica educativa del maestro/a, con la finalidad de proponer un modelo o secuencia metodológica para la aplicación del TP

\section{Factibilidad de la aplicación del TP en los diferentes niveles educativos y diferentes áreas de estudio.}

De acuerdo a los datos que arrojan los diferentes estudios revisados, el TP se puede aplicar ya sea en:

a) Educación Infantil y en áreas como la Educación Plástica y Visual, como fruto de la formación sistemática que proporciona la escuela; la materia artística requiere integrarse en un plan de trabajo no conductista, lo cual implica que la enseñanza no debe ser dirigida, sino que debe surgir a partir del alumnado para que así se desarrolle su pensamiento artístico (Gutiérrez, 2015).

b) Educación Secundaria, en áreas como la Educación Física, ya que el nivel de conocimiento aumenta si el alumno es partícipe de su propio aprendizaje. En este sentido, los autores concluyen que: i) si los aprendizajes responden a las necesidades, intereses y motivaciones del alumnado, éste incrementa el grado de adquisición de conocimientos; ii) cuando el alumno es un elemento activo dentro del proceso de enseñanza-aprendizaje, aumenta su interés y motivación; iii) si se utilizan metodologías innovadoras que favorecen la adquisición y contextualización de los aprendizajes hay una adquisición de conocimientos mayor; y, iv) cuando el alumno diseña su propio aprendizaje y conoce los objetivos, contenidos y criterios de evaluación, es más consciente del nivel de conocimiento (Aranda y García, 2016).

c) Educación Superior, por ejemplo, en asignaturas del Máster de Psicopedagogía de la Universidad Jaume I, cuyos investigadores se dieron cuenta de que el TP permite poner de manifiesto la renuncia al sentido excluyente de la universidad como contexto formativo, pues permite ampliar y enriquecer las experiencias de aprendizaje (Moliner y Sánchez-Tarazaga, 2015).

\section{Contribución del TP al éxito del proceso de enseñanza-aprendizaje}

Los estudios demuestran que los TP sirven de herramienta para investigar en diversos contextos educativos cuya necesidad sea establecer redes formativas entre profesionales en educación y/o crear comunidades de aprendizaje. Además, permiten la construcción de puentes entre la investigación, la reflexión y la práctica en las aulas, permitiendo la organización del currículum y la gestión del espacio y del tiempo (Mérida et al., 2011; Ventura, 2013).

En cuanto a la actualización de contenidos y las formas de enseñar a los niños, algunos estudios demuestran que tras la aplicabilidad del TP en las aulas, Ios niños/as aprenden a comunicarse, se favorecen las relaciones sociales, se afronta el desarrollo intelectual, personal e intrapersonal del alumnado, y se establecen valores, actitudes, competencias y habilidades útiles para la vida (Ureña, 2013).

Asimismo, es importante destacar el estudio de Ortega (2016), quien hace referencia a la importancia del TP en los cursos de formación e innovación del profesorado, debido a que permite abordar temas abiertos y actuales. En este sentido, Abad et al. (2015), añaden que este enfoque metodológico contribuye a la reflexión del docente al contrastar la realidad con el modelo ideal que promueve el TP. Por consiguiente, la formación académica debe tener en cuenta la condiciones teórico-prácticas de este enfoque, contribuyendo así a la formación de un grupo sólido de maestros/as que luego lo implementen en sus aulas.

Otro aspecto a considerar para el éxito del proceso de enseñanza, es la participación de la familia y la inclusión de la comunidad educativa. A través del TP se puede conseguir la participación de todos los sectores de la comunidad educativa. De esta manera, la heterogeneidad conlleva a que se multipliquen los aprendizajes (Mérida y Ruiz, 2015). 


\section{Principios metodológicos que fundamentan al TP.}

Según las investigaciones, el TP es un modelo alternativo al enfoque curricular tradicional, basado en unos principios pedagógicos fundamentados, coherentes y sólidos, derivados de diferentes fuentes y perspectivas. Estos principios, constituyen las bases ideológicas de la práctica educativa del docente o la manera de conectar la práctica cotidiana dentro de su aula y la teoría aprendida. (Mérida, et al., 2011; Ureña, 2013; Mérida y Ruiz, 2015; Ventura, 2013; Fernández, 2016). Esta hibridación de fundamentos en los que se sustenta, se muestra en la figura 1.

Figura 1. Principios metodológicos que fundamentan al TP.

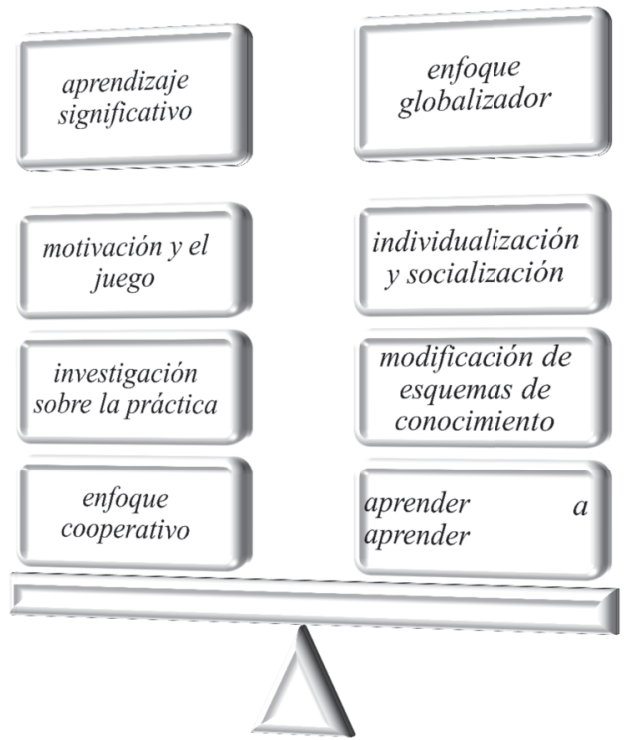

\section{Fuente: Elaboración propia}

Dentro de estos principios, cabe destacar el enfoque globalizador del TP, pues permite abordar todos los contenidos de las distintas áreas del currículum educativo.

Durante la revisión de estudios, se pudo evidenciar que, aunque parece que el TP ofrece muchas ventajas cualitativas en el proceso de enseñanza-aprendizaje, en las aulas hay muchos maestros que aún defienden la postura relacionada con una metodología tradicional. Es por ello que, antes de plantear algún modelo o secuencia metodológica para la aplicación del TP, surgieron tres interrogantes, factibles de convertirse en hipótesis para posteriores estudios:

1. Cuantos más años de experiencia tiene un/a maestro/a ¿tiende a atreverse más con las nuevas metodologías?

2. La formación que reciben los maestros/as ¿influye en su decisión respecto a la metodología utilizada? Y, si se les forma en nuevas metodologías, ¿tienden a utilizar éstas y no otras?

3. El uso de nuevas metodologías, como el TP, ¿implican un mayor esfuerzo y trabajo, por parte del maestro, que las metodologías tradicionales? 


\section{EL TRABAJO POR PROYECTOS EN EL CURRÍCULO EDUCATIVO: PRINCIPIOS METODOLÓGICOS QUE LO FUNDAMENTAN}

\section{Modelo o secuencia metodológica para la aplicación del TP}

Este método se basa en el aprendizaje significativo, en el trabajo cooperativo/ colaborativo y en la investigación por parte de los alumnos, basado en la relación de diferentes áreas de conocimiento. A partir de esta idea, se han revisado estudios que muestran alguna secuencia metodológica para su puesta en práctica. Sin embargo, antes de proponer algún modelo, conviene aclarar que en el desarrollo de cualquier proceso educativo no puede existir una receta metodológica válida para todos/as, puesto que la realidad de las distintas aulas es muy compleja y, lo que sucede dentro de ellas, dependerá de muchas variables: de las características de los diferentes agentes que intervienen en el proceso, de los espacios, de los materiales con los que se cuenta, de los horarios, del momento en el que tenga lugar dicho proceso educativo. Por ello, el principal objetivo no es solo conocer las características de las estrategias a diseñar, sino discernir lo que es más conveniente hacer a la hora de gestionar una clase.

Para el desarrollo de un TP, la mayoría de los estudios coinciden en seguir el procedimiento o fases de aplicación (no secuenciales), que se presentan en la figura 2 (Ureña, 2013; Abad et al., 2015).

Figura 2. Propuesta de fases para la aplicación del TP

Conocción del tema de estudio

Hay que insistir en que los momentos que aquí se presentan no son momentos rígidos ni estáticos, no obedecen a fases fijas y predeterminadas que hayan de seguirse en el desarrollo de un proyecto. Éste debe ser algo dinámico, en continua evolución y adaptado a los avatares y propuestas que vayan surgiendo en el grupo (Ureña, 2013; Abad et al., 2015).

Dentro del proceso, se responden a cuestiones tales como: ¿Qué objetivos generales se quieren conseguir con este proyecto?; ¿Qué objetivos didácticos se pretenden que los alumnos/as alcancen?; ¿Qué contenidos se van a trabajar?; ¿Qué actividades se plantean?; ¿Cómo implicar a las familias?; ¿Cómo realizar la evaluación?; ¿Qué instrumentos utilizar?

Asimismo, es importante recalcar que el TP son investigaciones realizadas en el aula por los propios discentes. Los temas a tratar, casi siempre, suelen surgir de los mismos discentes o de situaciones de aprendizaje creadas por los profesores. Se centran en temas de interés y cercanos a los discentes que hacen que muestren una predisposición e implicación mucho mayor, ya que ellos están totalmente sumergidos en lo que están aprendiendo y trabajando. Los proyectos no tienen una duración determinada, ya que la temporalización es flexible y adaptable a todas las situaciones que pueden surgir en el aula. Además, este tipo de metodología es interdisciplinar, 
son varias las asignaturas y áreas que están adquiriendo y aprendiendo al mismo tiempo. Éstas se integran unas con otras. El aprendizaje se construye entre todos, discentes y docentes, lo forman día a día, trabajando de manera colaborativa y cooperativa.

Para la evaluación de los proyectos, se toman en consideración aspectos intrínsecos al propio proyecto y aspectos de valoración de la aplicación práctica de los mismos.

Dentro de los aspectos intrínsecos, tal y como lo demuestran Aranda y García (2016), y como parte de la evaluación continua, se pueden considerar los que se encuentran en la figura 3.

Figura 3. Evaluación de aspectos intrínsecos al propio proyecto

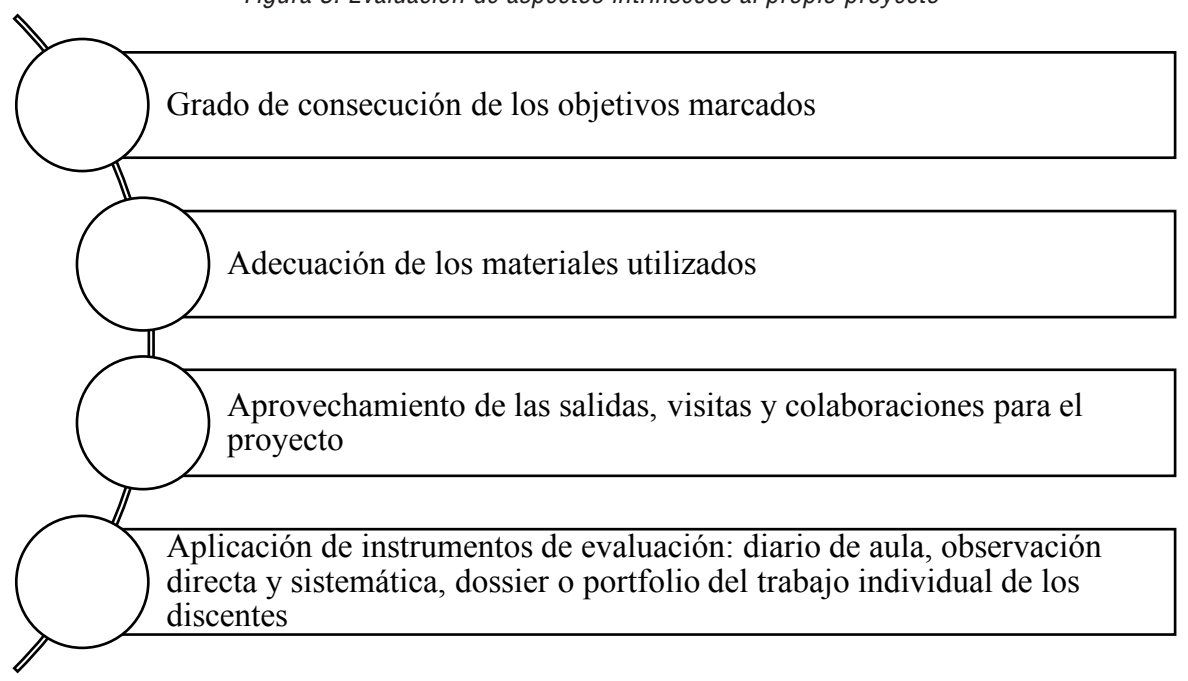

Para la valoración de la aplicación práctica o experiencia obtenida en el aula, los estudios muestran que se realiza a través de la descripción de la observación directa, tomando en consideración la propuesta de cinco criterios, basados en los principios metodológicos de los TP: globalización de los aprendizajes, observación, experimentación e investigación del alumnado, aprendizaje significativo, socialización y autoconcepto positivo, organización del trabajo alrededor de una problemática a investigar (Jato, 2015).

\section{DISCUSIÓN}

El análisis de los resultados obtenidos permite dar respuesta a uno de los planteamientos iniciales: ¿el TP es necesario y factible en la enseñanza actual? La respuesta a esta pregunta es sí, pues en todos los artículos recogidos, en mayor o menor medida, se encuentra que los autores coinciden tanto en la necesidad de estas nuevas metodologías, como en las muchas aportaciones positivas que éstas pueden hacer en la enseñanza.

En cuanto a las interrogantes que surgieron, a partir del tratamiento de la información obtenida en los estudios revisados, con miras a proponer algún modelo o secuencia metodológica para la aplicación práctica del TP, la información obtenida muestra que:

1) Ios docentes prefieren una metodología basada en proyectos, ya que beneficia las condiciones del alumnado en su contexto de aprendizaje y la construcción progresiva de sus conocimientos (Balcells, 2014).

2) la formación recibida por el profesorado influye a la hora de decidir qué metodologías emplear en el aula (Sanmartí, 2016).

3) es cierto que una metodología basada en proyectos exige mayor dedicación y esfuerzo en la acción docente. La diferencia, según los criterios de los estudios, es la implicación del alumnado en su propio proceso de 


\section{EL TRABAJO POR PROYECTOS EN EL CURRÍCULO EDUCATIVO: PRINCIPIOS METODOLÓGICOS QUE LO FUNDAMENTAN}

aprendizaje, visto como una reconstrucción de sus conocimientos con el maestro como guía (Abad et al., 2015; Orozco y Perochena, 2016; Gil y Rivero, 2014).

\section{CONCLUSIONES}

En vista de los resultados analizados, el TP es una metodología que es factible de ser aplicada en cualquier área, nivel o modalidad del sistema educativo. Su puesta en práctica para la gestión dentro del aula, de una manera íntegra, aporta contenidos significativos y necesarios para el éxito del alumnado.

Asimismo, aunque se es consciente de que trabajar por proyectos conlleva cierta implicación, por lo que el esfuerzo es mayor, al discernir qué método utilizar, el profesorado debe tener en cuenta aspectos como: Ios intereses del alumnado, sus capacidades, sus necesidades, el hecho de que sean partícipes de su propio proceso de aprendizaje u otras características de carácter didáctico y pedagógico que hacen que la educación sea de calidad.

\section{REFERENCIAS BIBLIOGRÁFICAS}

Abad Alonso, N., Fuentes Abeledo, E., y Muñoz Carril, P. (2015). De los proyectos de trabajo soñados a la realidad del aula en Educación Infantil. Revista de Estudios e Investigación en Psicología y Educación, O(6). DOI: 10.17979/reipe.2015.0.06.349.

Aranda, P. y García, C. (2016). El Aprendizaje basado en Proyectos en el área de educación física. Actividad Física Y Deporte: Ciencia Y Profesión., (24), 53-66. Recuperado de http://colefcafecv.com/wpcontent/uploads/2016/09/art4.24.pdf

Balcells, M. (2014). El trabajo por proyectos: una metodología global. Madrid: Cuadernos de Pedagogía, Wolters Kluwer, No. 450.

Fernández, S. (2016). Aprendizaje Basado en Proyectos. El qué, el cómo y la evaluación. Recuperado de: http://www.educaciontrespuntocero.com/opinion/aprendizaje-basado-proyectos-la-evaluacion/36839.html

Gil Alejandre, J. y Rivero García, P. (2014). Somos romanos. Análisis de una experiencia de trabajo por proyectos en Educación Infantil. History and history Teaching. Universidad de Zaragoza.

Gutiérrez, R. (2015). El Trabajo por Proyectos para la construcción compartida del currículum de artes plásticas y visuales en la formación del profesorado de educación infantil. Ensayos. Revista De La Facultad De Educación De Albacete, 30(2), 59-269. Recuperado de http://www.revista.uclm.es/index.php/ensayos.

Jato, A. (2015). ¡Cuánto sabemos! El día a día en el Trabajo por Proyectos. Madrid: Santillana.

Mérida, R., Barranco, B., Criado, E., Fernández, N., López, R.M. y Pérez, I. (2011). Aprender investigando en la escuela y la universidad. Una experiencia de investigación- acción a partir del Trabajo por Proyectos. Investigación en la escuela, 73, 65-76.

Mérida Serrano, R. y Ruiz Torres, M. C. (2016). Promover la inclusión de las familias a través del desarrollo de Proyectos de Trabajo. Un estudio de caso. Revista Complutense de Educación, 27(3), 943-961. D0I: 10.5209/rev_RCED. 2016.V27. N3.47022.

Moliner, 0. y Sánchez-Tarazaga, L. (2015). PBL o Aprendizaje basado en Proyectos: una experiencia de coordinación y enseñanza auténtica en la universidad. Quadernsdigitals.Net, 81, 116-133. Recuperado de http://www.quadernsdigitals.net/index.php?accionMenu=hemeroteca.VisualizaArticulolU.visualiza\&articuI0_id=11426

Ortega, P. (2016). Los trabajos por proyectos en la Educación Infantil. En primera persona: estudio de caso biográfico-narrativo de una maestra. Investigación en la Escuela, 88, 112-126. Recuperado de www.investigacionenlaescuela.es/artiuclos/R88/R88-7

Orozco Moreno, V. y Perochena González, P. (2016). Trabajo por proyectos. Cambio metodológico para el protagonismo del estudiante en Educación Infantil (2-3 años). Revista De Estudios Y Experiencias En Educación, 15(29), 151-164. 
Pacheco Sanz, D. I., Canedo-García, A., Carpio Pacheco, D. y García Sánchez, J.N. (2019). Posibilidades y limitaciones de las metodologías didácticas basadas en las inteligencias múltiples. En D. Padilla Góngora, J.M. Aguilar Parra y R. López Liria (Eds.) Salud y Ciclo Vital (pp. 297-308). Madrid: Dykinson.

Sanmartí, N. (2016). Trabajo por proyectos: ¿Filosofía o metodología? Cuadernos de Pedagogía, 472, 1-6.

Ureña Borrego, M. (2013). Competencias y proyectos en Educación Infantil: hacia una educación holística. Calanda: Revista didáctica de la acción educativa española en Francia, (8), 22-31.

Ventura, M. (2013). La perspectiva de los proyectos de trabajo como un motor de cambio en la organización del currículum y en la gestión del tiempo y el espacio. Investigación en la escuela, 79, 19-30. 
